\title{
Neurofibromin C terminus-specific antibody (clone NFC) is a valuable tool for the identification of NF1-inactivated GISTs
}

Sabrina Rossi ${ }^{1,8}$, Daniela Gasparotto ${ }^{2,8}$, Matilde Cacciatore ${ }^{1}$, Marta Sbaraglia ${ }^{1}$, Alessia Mondello ${ }^{2}$, Maurizio Polano ${ }^{2}$, Alessandra Mandolesi ${ }^{3}$, Alessandro Gronchi ${ }^{4}$, David E Reuss 5,6 , Andreas von Deimling ${ }^{5,6}$, Roberta Maestro ${ }^{2,9}$ and Angelo Paolo Dei Tos ${ }^{1,7,9}$

${ }^{1}$ Department of Pathology \& Molecular Genetics, Treviso General Hospital, Treviso, Italy; ${ }^{2}$ Oncogenetics and Functional Oncogenomics Unit, CRO Aviano National Cancer Institute, Aviano, Italy; ${ }^{3}$ Department of Internal Medicine, Ospedali Riuniti di Ancona, Ancona, Italy; ${ }^{4}$ Department of Surgery, Fondazione IRCCS Istituto Nazionale dei Tumori di Milano, Milano, Italy; ${ }^{5}$ Department of Neuropathology, Institute of Pathology, University of Heidelberg, Heidelberg, Germany; ${ }^{6}$ Clinical Cooperation Unit Neuropathology, German Cancer Consortium (DKTK), German Cancer Research Center (DKFZ), Heidelberg, Germany and ${ }^{7}$ Department of Medicine, University of Padova School of Medicine, Padova, Italy

An increasing body of evidence supports the involvement of NF1 mutations, constitutional or somatic, in the pathogenesis of gastrointestinal stromal tumors (GISTs). Due to the large size of the NF1 locus, the existence of multiple pseudogenes and the wide spectrum of mechanisms of gene inactivation, the analysis of NF1 gene status is still challenging for most laboratories. Here we sought to assess the efficacy of a recently developed neurofibromin-specific antibody (NFC) in detecting NF1-inactivated GISTs. NFC reactivity was analyzed in a series of 98 GISTs. Of these, 29 were 'NF1-associated' (17 with ascertained NF1 mutations and 12 arising in the context of clinically diagnosed Neurofibromatosis type 1 syndrome and thus considered bona fine NF1 inactivated); 38 were 'NF1-unrelated' (either wild-type or carrying non-pathogenic variants of NF1). Thirty-one additional GISTs with no available information on NF1 gene status or with NF1 gene variants of uncertain pathogenic significance were also included in the analysis. Cases were scored as NFC negative when, in the presence of NFC positive internal controls, no cytoplasmic staining was detected in the neoplastic cells. NFC immunoreactivity was lost in $24 / 29(83 \%)$ NF1-associated GISTs as opposed to only $2 / 38(5 \%)$ NF1-unrelated GISTs $(P=3 e-11)$. NFC staining loss significantly correlated $(P=0.007)$ with the presence of biallelic $N F 1$ inactivation, due essentially to large deletions or truncating mutations. NFC reactivity was instead retained in two cases in which the NF1 alteration was heterozygous and in one case where the pathogenic NF1 variant, although homo/hemizygous, was a missense mutation predicted not to affect neurofibromin half-life. Overall this study provides evidence that NFC is a valuable tool for identifying NF1-inactivated GISTs, thus serving as a surrogate for molecular analysis.

Modern Pathology (2018) 31, 160-168; doi:10.1038/modpathol.2017.105; published online 1 September 2017

Neurofibromin, encoded by the NF1 gene, is a GTPase-activating protein with tumor suppressor functions: neurofibromin binds and inhibits active

Correspondence: Dr R Maestro, PhD, Oncogenetics and Functional Oncogenomics Unit, CRO Aviano National Cancer Institute, Via Gallini 2, Aviano 33081, Italy or Professor AP Dei Tos, MD, Department of Pathology \& Molecular Genetics, Treviso General Hospital, Treviso 31100, Italy.

E-mail: maestro@cro.it or angelopaolo.deitos@aulss2.veneto.it

${ }^{8} \mathrm{~S}$ Rossi and D Gasparotto are co-first authors.

${ }^{9} \mathrm{R}$ Maestro and AP Dei Tos are co-last authors

Received 19 April 2017; revised 30 June 2017; accepted 30 June 2017; published online 1 September 2017
GTP-bound Ras, preventing the downstream triggering of Ras effector pathways (MAPK and PI3K/ Akt/mTOR). NF1 gene inactivation is typically involved in the pathogenesis of tumors arising in the context of Neurofibromatosis type 1, most notably malignant peripheral nerve sheath tumors, neurofibromas, and optic pathway gliomas. However, recent evidence has broadened the spectrum of NF1-driven tumors and NF1 mutations have been shown to contribute to the pathogenesis of over $10 \%$ sporadic tumors, including melanomas, glioblastomas, breast, ovarian and lung carcinomas, and sarcomas. ${ }^{1-4}$ 
We have recently reported that NF1 is a driver mutation in a significant fraction of gastrointestinal stromal tumors (GISTs). ${ }^{5}$ GIST is the most frequent mesenchymal neoplasm of the digestive tract. The vast majority of GISTs (about 85\%) are typically driven by activating mutations of receptor tyrosine kinases, namely KIT and PDGFRA. BRAF or HRAS mutations have been reported in a few cases. ${ }^{6,7}$ All these alterations result in the constitutive activation of MAPK and PI3K/Akt/mTOR pathways. About $5 \%$ of GISTs feature inactivation of components of the mitochondrial succinate dehydrogenase complex $(S D H)$ and are typically associated with syndromic conditions (Carney Stratakis and Carney Triad). The remaining fraction of GISTs (a.k.a. quadruple-negative GISTs, about $10 \%$ ) were essentially considered to be 'driver mutation unknown' until very recent literature highlighted that NF1 mutations play a greater role in GIST pathogenesis than commonly thought. ${ }^{4,5,8}$ NF1 mutations were historically considered to be confined to GISTs arising in the context of Neurofibromatosis type $1 .^{7,9}$ We instead demonstrated that NF1 mutations, be they constitutional or somatic, occur in a large proportion of 'apparently sporadic' quadruple-negative GISTs. ${ }^{5}$ The presence of NF1 mutations in GISTs has important diagnostic and predictive implications for patient management. Detection of an NF1-mutated GIST may be the sentinel of an unrecognized Neurofibromatosis type 1 condition, with obvious implications for the patient and his/her family. On the other hand, unlike receptor tyrosine kinase-mutated GISTs, which generally respond to kinase inhibitors, NF1-mutated GISTs are imatinib resistant. ${ }^{9,10}$ Hence, the detection of NF1 inactivation is crucial for proper clinical management of GIST patients.

The analysis of NF1 gene status is problematic for most laboratories and the diagnosis of Neurofibromatosis type 1 is still essentially based on clinical findings. The large size of the NF1 locus (58 exons and very large introns), the existence of multiple pseudogenes and the wide spectrum of mechanisms of gene inactivation (from missense mutations to large deletions, translocations and possibly epigenetic silencing), has long prevented comprehensive molecular testing of $N F 1$ and this investigation is still limited to a few laboratories equipped with massive parallel sequencing (next generation), comparative genomic hybridization technologies and multiplex ligation-dependent probe amplification approaches. ${ }^{1,11}$

Antibodies have proven to be reliable surrogates for gene alteration analysis in different contexts. For instance, HER2 staining is used to identify HER2amplified breast cancers; anti-MDM2 antibodies are used to identify MDM2-amplified liposarcomas; ALK immunoreactivity is indicative of $A L K$ rearrangements; ${ }^{12}$ loss of SDHB staining is considered indicative of $S D H x$ gene inactivation, ${ }^{13}$ and VE1 antibody is a good surrogate for BRAF V600E mutation detection, also in the context of GISTs. ${ }^{14-16}$
We recently generated an antibody, NFC, directed against the last 281 amino acids of neurofibromin. ${ }^{17}$ This region is commonly lost in NF1-inactivated tumors, as the vast majority of NF1 mutations are deletions or give rise to truncated proteins. ${ }^{1}$ NFC has demonstrated high sensitivity and specificity in distinguishing NF1-associated malignant peripheral nerve sheath tumors from other spindle cell neoplasms. ${ }^{17,18}$

Here we sought to explore the reliability of NFC staining in identifying NF1-associated GISTs. To this end, we screened a well-characterized series of GISTs and correlated NFC reactivity with NF1 mutation data.

\section{Materials and methods}

\section{Tumor Samples}

Clinicopathological and molecular features of the analyzed tumor series are summarized in Table 1. The study was carried out on 98 formalin-fixed paraffin-embedded tumors retrieved from the pathological files of the collaborating institutions or submitted for consultation to one of the authors (APDT). The tumors-93 GISTs and 5 microGISTs (mGISTs, size $\leq 1 \mathrm{~cm}$ )—were from 89 patients (multiple tumors were analyzed for six patients). Twelve of these patients had a clinical diagnosis of Neurofibromatosis type 1 . Informed consent was obtained from all living individuals.

Paraffin block age ranged from 6 to 254 months (median 48 months). All samples were freshly cut prior to staining.

All 98 cases included in the study were profiled for KIT, PDGFRA, BRAF, or SDH gene status; one quadruple-negative GIST carried an ETV6-NTRK3 gene fusion. ${ }^{19}$ The NF1 gene status was ascertained in 56 samples: NF1 pathogenic mutations were detected in 17 tumors, all of them quadruplenegative; three cases-two KIT-mutated tumors (\#38, 39) and one quadruple-negative GIST (\# 98)bore non-pathogenic mutations and a genetic variant of uncertain pathogenic significance, respectively; 36 tumors were NF1 wild-type.

The nature of the NF1 mutations was assessed in 19/20 cases: the mutation was somatic in 4 GISTs (\#5, \#8, \#10, \#17) and constitutional in 15 (13 pathogenic, 1 non-pathogenic, and 1 variant of uncertain significance).

The five mGISTs included in the study were from patients with a diagnosis of Neurofibromatosis type 1.

The remaining 42 cases were not analyzed for NF1 status. These included 30 sporadic GISTs and 12 GISTs that were considered bona fide NF1 inactivated, having arisen in the context of clinically diagnosed Neurofibromatosis type 1.

Overall, from a molecular standpoint, our series was composed of three major groups: 'NF1-associated' 
Table 1 Clinicopathological and molecular characteristics of the series

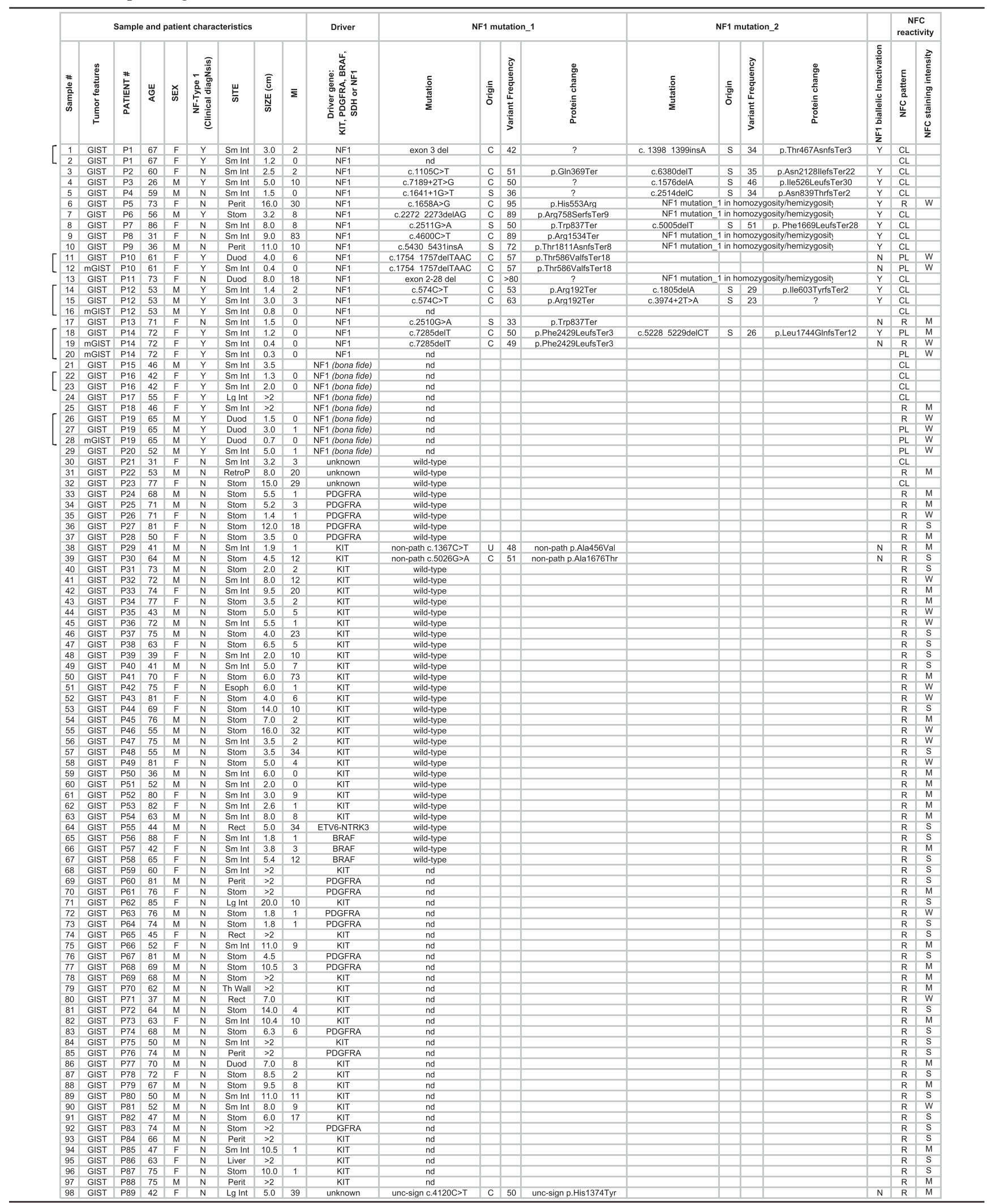

Abbreviations: CL, complete loss; Duod, duodenum; Esoph, esophagus; Lg Int, large intestine; M, moderate staining intensity; N, No; ND, NF1 mutation analysis not done; non-path, non-pathogenic NF1 variants; Perit, peritoneum; PL, partial loss; R, retained; Rect, rectum; RetroP, retroperituneum; S, strong staining intensity; Sm Int, small intestine; Stom, stomach; Th Wall, thoracic wall; U, unspecified; unc-sign, NF1 variant of uncertain pathogenic significance; W, weak staining intensity; Y, Yes.

Brackets indicate multiple tumors of the same patients. 
Table 2 NFC reactivity according to NF1 association

\begin{tabular}{|c|c|c|c|c|c|}
\hline NF1 association & $\begin{array}{l}\text { Driver mutation } \\
\text { (number of samples) }\end{array}$ & Evidence & NFC reactivity & NFC pattern of loss & $\begin{array}{l}\text { Number } \\
\text { of cases }\end{array}$ \\
\hline \multirow[t]{6}{*}{ NF1-associated } & Bona fide NF1 (12) & $\begin{array}{l}\text { Clinical diagnosis of neurofibromatosis } \\
\text { type } 1\end{array}$ & 10 Loss & 6 Complete, 4 partial & 29 \\
\hline & & & 2 Retention & No loss & \\
\hline & NF1 (17) & $\begin{array}{l}\text { Constitutional NF1 pathogenic } \\
\text { mutations (13 cases) }\end{array}$ & 11 Loss & 8 Complete, 3 partial & \\
\hline & & & 2 Retention & No loss & \\
\hline & & $\begin{array}{l}\text { Somatic NF1 pathogenic mutations } \\
\text { (4 cases) }\end{array}$ & 3 Loss & 3 Complete & \\
\hline & & & 1 Retention & No loss & \\
\hline \multirow[t]{4}{*}{ NF1-unrelated } & KIT (26) & NF1 wild-type or with non-pathogenic & 2 Loss & 2 Complete & 38 \\
\hline & PDGFRA (5) & $N F 1$ variants & & & \\
\hline & $\begin{array}{l}\text { BRAF (3) } \\
\text { ETV6-NTRK3 (1) }\end{array}$ & $\begin{array}{l}\text { No clinical evidence of } \\
\text { neurofibromatosis type } 1\end{array}$ & 36 Retention & No loss & \\
\hline & Unknown (3) & & & & \\
\hline \multirow[t]{4}{*}{ Undetermined } & KIT (21) & No information on NF1 gene status or & 31 Retention & No loss & 31 \\
\hline & PDGFRA (9) & $N F 1$ variants of uncertain pathogenic & & & \\
\hline & Unknown (1) & significance & & & \\
\hline & & $\begin{array}{l}\text { No clinical evidence of } \\
\text { neurofibromatosis type } 1\end{array}$ & & & \\
\hline
\end{tabular}

tumors (29 cases), including the 17 tumors carrying NF1 pathogenic mutations, together with the 12 GISTs arising in the context of clinically diagnosed Neurofibromatosis type 1; 'NF1-unrelated' tumors, consisting of the 38 cases with no or non-pathogenic NF1 mutations; 'NF1-undetermined' cases (31 cases), including the sporadic GISTs with no available information on NF1 status and the GIST (\# 98) carrying an NF1 variant of uncertain pathogenic significance (Table 2).

Irrespective of whether the origin of NF1 mutation was constitutional or somatic, the small intestine was the prevalent site in NF1-associated GISTs (20/29 cases), as different from the NF1-unrelated tumors (16/38 cases) $(P=0.047)$, where gastric lesions were instead more common $(19 / 38)(P=3 e-5)$ (Table 1$)$, in agreement with previous reports. . $^{50,21}$

\section{Immunohistochemistry}

The NFC monoclonal antibody was used to assess neurofibromin protein expression by immunohistochemistry. This antibody was raised in mice by immunization with a recombinant protein corresponding to the $\mathrm{C}$ terminus of human neurofibromin (last 281 amino acids), a region commonly lost in NF1-inactivated tumors. ${ }^{17} \mathrm{NFC}$ demonstrated high sensitivity and specificity in the identification of NF1-associated malignant peripheral nerve sheath tumors. ${ }^{17}$

NFC immunostaining was performed on whole sections of the 29 NF1-associated tumors listed in Table 2 plus the case carrying an NF1 variant of uncertain significance. Tissue microarrays were implemented for the remaining cases. Each tumor was represented by three 2-mm cores.

Freshly cut $4 \mu \mathrm{m}$ sections were first dried at $60^{\circ} \mathrm{C}$ for $30 \mathrm{~min}$ and then stained using an automated immunostainer (Dako AutostainerLink 48). The staining was preceded by heat-induced antigen retrieval $\left(98^{\circ} \mathrm{C}\right.$, waterbath) carried out in citrate buffer, pH 6.1 for 40 min (EnVision FLEX Target Retrieval Solution, low $\mathrm{pH}$ ). The DAKO staining procedure included 5 min' incubation with FLEX Peroxidase Block, $1 \mathrm{~h}$ of incubation with 1:4 diluted NFC hybridoma supernatant, 15 min incubation with EnVision FLEX+ Mouse LINKER (Dako), 20 min incubation with the labeled polymer (HT/HRP), and $10 \mathrm{~min}$ incubation with a substrate chromogen (FLEX DAB+Sub Chromo). All staining steps were carried out at room temperature.

NFC expression was considered lost when no cytoplasmic staining was seen in the neoplastic cells but appropriate internal controls (fibroblasts, ganglion cells, plasma cells, endothelium, smooth muscle) reacted positively. NFC loss was regarded as complete when the neoplastic cells were homogeneously negative, and partial when the staining was heterogeneous with limited tumor areas retaining NFC expression. NFC expression was considered to be maintained when tumor cells were homogeneously positive. Staining intensity (weak, moderate, strong) was recorded.

\section{Molecular Analysis}

For the purposes of molecular analysis, areas enriched in tumor cells were marked by the pathologist on tissue slides. DNA was extracted with the EZ1 biorobot (Qiagen) or QIAamp DNA FFPE Tissue kit (Qiagen). Massive parallel sequencing libraries were prepared with a TruSeq Custom Amplicon v1.5 panel (Illumina), run on MiSeq (Illumina), and analyzed as previously described. ${ }^{5}$ NF1 mutations were validated by Sanger sequencing (Reference NF1 gene sequence: NM_001042492.2; Reference neurofibromin protein sequence: NP_001035957.1). Regions with low coverage at massive parallel sequencing analysis were also 
double checked by Sanger sequencing on an ABI PRISM 3100 Genetic Analyzer (Applied Biosystems). Only variants with frequencies $>20 \%$ were considered.

Mutations were considered heterozygous when the frequency was in the $20-60 \%$ range; homo/hemizygous when it was $>70 \%$. NF1 biallelic inactivation was defined by the presence of a homo/ hemizygous mutation or by two distinct heterozygous NF1 mutations in the same tumor. Where available, matched normal DNA was analyzed to determine whether the mutation detected in the tumor was constitutional or somatic.

The Human Gene Mutation Database (http://www. hgmd.cf.ac.uk) and ClinVar (https://www.ncbi.nlm. nih.gov/clinvar) were interrogated to search for published associations with the Neurofibromatosis type 1 syndrome of the identified mutations. In addition, Provean (http://provean.jcvi.org), SIFT (http://sift.bii.a-star.edu.sg), PolyPhen2 (genetics.bwh. harvard.edu/pph2), MutationAssessor (http://mutation assessor.org/), and PaPI (http://papi.unipv.it) tools were used to predict the effect of non-synonymous variants. The Human Splicing Finder software (www. umd.be/HSF3) was used to evaluate splice site mutations. The ExPASy ProtParam tool (http://web. expasy.org/protparam/) was implemented to assess the impact of single amino-acid variations on protein half-life.

\section{Statistical Analysis}

Fisher's exact test (two-tailed) was used to compare categorical variables.

\section{Results}

The results of NFC immunostaining are summarized in Tables 1 and 2. Representative samples are shown in Figure 1. Clear-cut positive internal controls were always present in all analyzed cases.

NFC staining was cytoplasmic and granular in quality in both neoplastic and non-neoplastic/positive control cells. Ganglion cells and nerves, followed by plasma cells and fibroblasts, displayed the strongest reactivity. Endothelial cells showed highly variable staining intensity (from moderate to weak). Regarding the gastric mucosa, NFC reactivity was positive in the glands, usually with moderate intensity; the foveolae were instead negative. NFC stained the intestinal mucosa homogeneously but with variable intensity. NFC reactivity in the smooth muscle cells of the muscularis propria was usually weak.

Loss of NFC immunostaining was observed in 26 of the 98 analyzed tumors, specifically in 24/29 (83\%) NF1-associated and 2/38 (5\%) NF1-unrelated GISTs $(P=3 \mathrm{e}-11)$. Among the $24 N F 1$-associated tumors displaying loss of NFC reactivity (20 GISTs and 4 mGISTs), 17 samples showed complete loss (16 GISTs and 1 mGISTs) and 7 partial loss (4 GISTs and 3 mGISTs) (Figures 1a-d). Specifically, NFC deficiency was detected in 11/13 tumors with constitutional pathogenic NF1 mutations; 10/12 tumors arising in clinically diagnosed Neurofibromatosis type 1 patients; and 3/4 tumors with somatic pathogenic NF1 mutations.

Of the NF1-unrelated tumors, loss of NFC reactivity (complete loss) was observed in only 2/38 GISTs (\#30 and \#32 in Table 1; Figure 1e). Both these tumors were positive for CD117 and DOG1. One was a low-risk GIST with mixed morphology arising in the small intestine; the other was a localized high-risk GIST with spindle morphology occurring in the stomach. These patients (a 31-year-old woman and a 77-year-old lady), who presented no diagnostic signs of Neurofibromatosis type 1, did not receive any adjuvant therapy and were disease free at the last follow-up (13 and 25 months, respectively). Due to the absence of mutations in KIT, PDGFRA, RAS $(\mathrm{H} / \mathrm{K} / \mathrm{N}), B R A F, S D H$, and $N F 1$ genes, these two GISTs were classified as 'driver mutation unknown'.

Loss of NFC reactivity correlated with biallelic inactivation of NF1 $(P=0.007)$, due to either large deletions or truncating mutations.

NF1 gene status was known for three of the five NF1-associated tumors retaining NFC reactivity: sample \#6 was a GIST with weak NFC staining intensity carrying a homo/hemizygous missense mutation (His553Arg) (Figure 1f). According to the ExPASy ProtParam tool, this variation does not significantly impact on protein turnover. The other two samples, featuring weak (\#17) or moderate (\#19) NFC reactivity, were both small tumors (size $<1.5 \mathrm{~cm}$ ) that retained a wild-type $N F 1$ allele besides the one carrying a truncating mutation.

Another GIST (\#98) carried the heterozygous NF1 mutation His1374Tyr. This missense variant was classified by ClinVar to be of uncertain pathogenic significance, which is why this GIST was included in the group of tumors with an unknown driver gene and undetermined NF1 association. In this case, too,

Figure 1 (a) Case \#9: NF1-associated GIST showing complete NFC loss. Fibroblasts were used as internal positive controls (magnification $\times 20$ ). (b) Case \#1: NF1-associated GIST showing complete NFC loss. Inflammatory cells served as internal positive controls (magnification $\times 40$ ). (c) Case \# 22: NF1-associated GIST showing complete NFC loss. Weak reactivity is present in the endothelium (positive control) (magnification $\times 40$ ). (d) Case \#28: NF1-associated microGIST showing partial loss of NFC (magnification $\times 10)$. (e) Case \#30: complete NFC loss in an NF1 wild-type GIST. This case was considered as 'driver gene unknown', being wild-type not only for NF1 but also for KIT, PDGFRA, RAS (H/K/N), BRAF, and SDHx. Normal vessels served as internal positive controls (magnification $\times 20$ ). (f) Case \#6: NF1associated GIST showing weak NFC reactivity. This case carried a homo/hemizygous missense mutation at the $\mathrm{N}$ terminus (His553Arg) (magnification $\times 40)$. (g, h) Two NF1 'wild-type'/KIT-driven GISTs (\#46, \#57) showing strong NFC reactivity (magnification $\times 40$ ). 

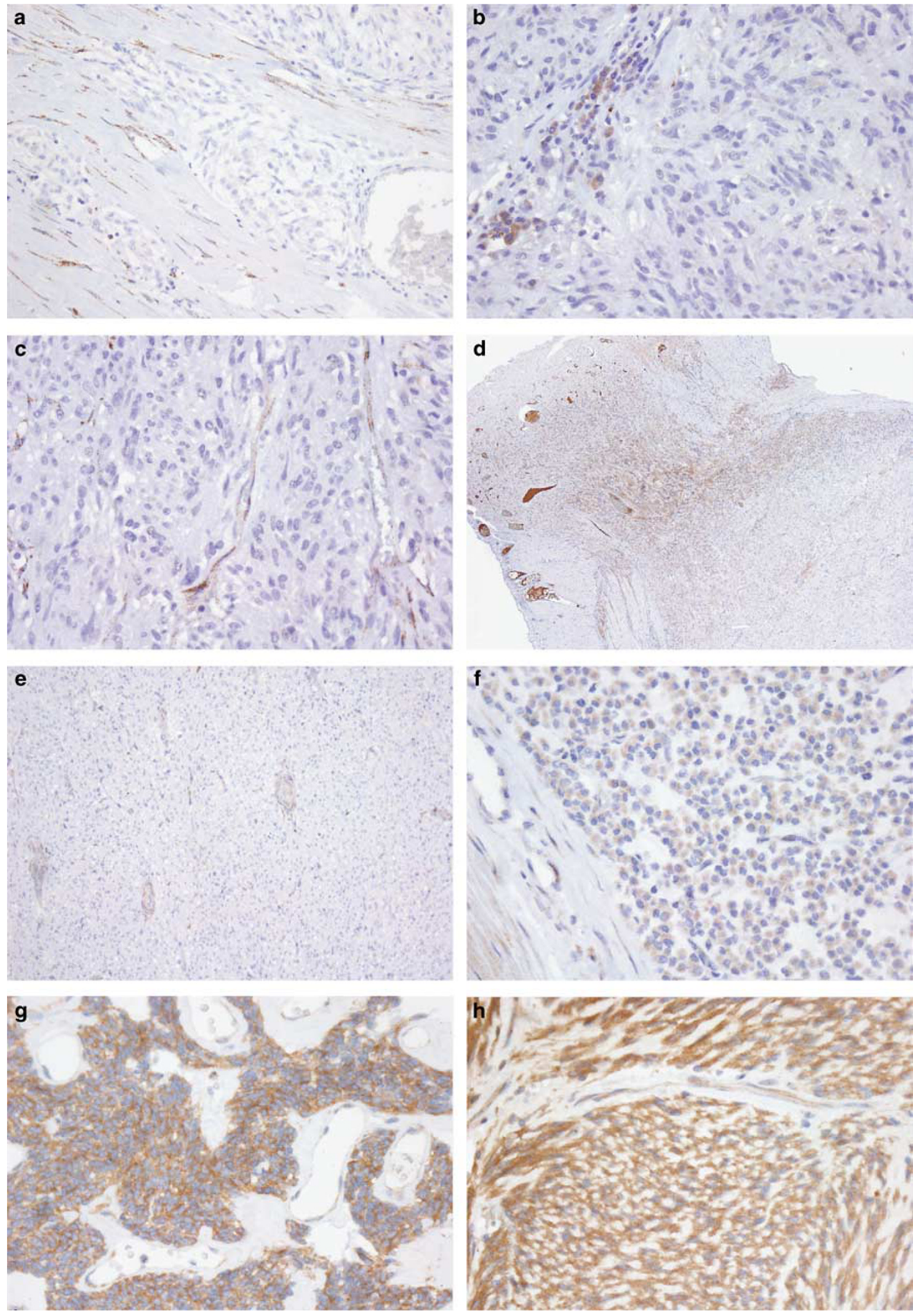
the tumor retained a wild-type allele and maintained NFC reactivity.

The NFC reactivity retained in 36/38 NF1 wildtype tumors was strong (12 samples) or moderate (15 samples) in most cases (Figures $1 \mathrm{~g}$ and h). Weak staining was detected in 9 samples that were relatively old ( $\geq 4$ years), indicating that pre-analytical factors can influence NFC performance, as previously described. ${ }^{17}$

No information on NF1 gene status was available in 30 samples. This set included 21 KIT- and 9 PDGFRA-driven GISTs. All these tumors retained NFC reactivity (strong in 17 samples, moderate in 11 and weak in 2).

\section{Discussion}

We have recently demonstrated that NF1 mutations play a prominent role in the pathogenesis of apparently 'sporadic' GISTs. ${ }^{5}$ Specifically, we have reported that about $60 \%$ of so-called quadruplenegative GISTs (GISTs lacking KIT, PDGFRA, BRAF, and $S D H$ mutations; $10 \%$ of all GISTs) carry NF1 mutations that are constitutional in most cases. Our work has thus demonstrated that a significant fraction of quadruple-negative GISTs arise in the context of unrecognized Neurofibromatosis type 1 syndrome. The identification of NF1-driven GISTs has important clinical implications, both in terms of patient and family surveillance, due to their cancerprone condition; and in terms of treatment strategies, considering the poor sensitivity to imatinib of NF1-related tumors. ${ }^{9,10}$ In this regard, the promising results recently obtained with MEK inhibitors in the treatment of unresectable NF1-associated plexiform neurofibromas ${ }^{22}$ open novel therapeutic opportunities for NF1-associated GISTs.

Unfortunately, the detection of NF1 mutations, be they constitutional or somatic, is challenging due to the large size of the gene and the numerous mechanisms of inactivation. ${ }^{1,11}$

On these grounds, we sought to explore the efficacy of an immunohistochemical approach to identifying NF1-mutated GISTs. To this end, we collected a series of NF1-associated GISTs (including both GISTs and mGISTs arising in the context of clinically diagnosed Neurofibromatosis type 1 and NF1mutated tumors submitted to the pathologist as 'sporadic') and NF1-unrelated tumors, and interrogated the cohort with the NFC antibody, a customraised antibody directed against the $\mathrm{C}$ terminus of neurofibromin. This antibody has previously been shown to differentiate malignant peripheral nerve sheath tumors, typically NF1-associated neoplasms, from other NF1-unrelated mimics. ${ }^{17,18}$

The results of our analysis can be summarized as follows: the vast majority (83\%) of the tested NF1-associated GISTs/mGISTs displayed complete or partial loss of NFC reactivity. Staining successfully identified 12 of the 13 tumors carrying homo/ hemizygous NF1 gene inactivation due to large deletions or truncating mutations, irrespective of whether the alteration was of somatic or constitutional origin. There was one exception: a case (\#6) bearing a homo/hemizygous N-terminal His553Arg pathogenic missense mutation. Said mutation was predicted by ProtParam to not significantly impact on neurofibromin turnover. This finding corroborates previous observations that NFC reactivity is likely preserved by stable neurofibromin variants. ${ }^{17}$

The inability to reveal missense mutations is an intrinsic drawback of antibodies designed to distinguish full length vs truncated proteins (eg, antibodies against X-linked alpha-thalassemia/mental retardation syndrome, ATRX). ${ }^{23,24}$ However, $<10 \%$ of NF1 pathogenic mutations reported in the ClinVar database are missense mutations. ${ }^{25}$

NFC staining was retained not only in the two GISTs carrying non-pathogenic missense mutations (\#38 and \#39), but also in the two tumors (\#17, \#19) that retained a wild-type NF1 allele, while carrying truncating mutations. This suggests that the amount of neurofibromin encoded by a single wild-type allele is sufficient to yield NFC reactivity.

In NF1-unrelated GISTs, NFC reactivity was retained in all but two tumors (95\%). Notably, these two tumors were also negative for canonical KIT, PDGFRA, BRAF, and SDHx mutations. Despite the lack of NF1 mutations, their negativity for NFC may underlie the existence of alternative mechanisms of NF1 inactivation. For instance, it has been demonstrated that enhanced proteasomal destruction accounts for NF1 loss in glioblastomas. ${ }^{26,27}$ Thus, NF1 could play an even greater role in the pathogenesis of GISTs than inferred by gene mutation analysis. ${ }^{5}$

As regards the pattern of staining, NFC loss was complete in most cases (17 cases). Partial loss was observed in seven NF1-associated tumors in which NFC reactivity was retained in focal areas. Partial loss has previously been reported to occur in malignant peripheral nerve sheath tumors ${ }^{17}$ and could be ascribed to tumor heterogeneity. Intriguingly, partial loss was more common among microGISTs-considered to be bona fide GIST precursors $^{6,28}$ - than among overt GISTs. This suggests that full inactivation of NF1 contributes to microGIST progression to overt GIST.

From a technical standpoint, although our series consisted of archival material, a clear-cut diffuse staining pattern of remarkable intensity was observed in the majority of NFC-positive cases, while weak reactivity was essentially observed in older samples ( $\geq 4$ years).

As previously discussed, ${ }^{17}$ similarly to other tumor suppressors, the interpretation of neurofibromin staining also relies on robust internal positive controls. Loss of staining for INI1, ATRX, SDHB, and SDHA, which are ubiquitously expressed, is not usually an issue as internal positive controls are easy to find. ${ }^{12,13,23,24,29}$ Neurofibromin has been reported 
to be expressed at high levels in all fetal rat tissues, but is essentially confined to the central and peripheral nervous systems postnatally. ${ }^{30,31}$ Accordingly, we observed strong NFC reactivity in ganglion cells and in the myenteric plexus; fibroblasts and inflammatory cells could also be positive, thus serving as additional internal controls. By contrast, the endothelium tended to display weak NFC staining, which could be overlooked at low power.

Overall our results provide evidence that NFC staining is a simple, rapid, and cost effective strategy for identifying NF1-inactivated GISTs, thus constituting a surrogate for molecular analysis. In addition, this study adds further support to the relevance of NF1 in the inception and progression of GISTs.

\section{Acknowledgments}

This work was supported by a CRO Intramural Grant and by the Italian Ministry of Health (RF-201102348953). We are grateful to M. Mafra, $P$. Iuzzolino, A. Rizzo for their support and suggestions.

\section{Disclosure/conflict of interest}

AG and APDT have received honoraria and participated in Compensated Advisory Boards for Novartis and Pfizer. AG has also participated in Compensated Advisory Boards for Bayer. All remaining authors have declared no conflict of interest.

\section{References}

1 Ratner N, Miller SJ. A RASopathy gene commonly mutated in cancer: the neurofibromatosis type 1 tumour suppressor. Nat Rev Cancer 2015;15:290-301.

2 Krauthammer M, Kong Y, Bacchiocchi A, et al. Exome sequencing identifies recurrent mutations in NF1 and RASopathy genes in sun-exposed melanomas. Nat Genet 2015;47:996-1002.

3 Hechtman JF, Zehir A, Mitchell T, et al. Novel oncogene and tumor suppressor mutations in KIT and PDGFRA wild type gastrointestinal stromal tumors revealed by next generation sequencing. Genes Chromosomes Cancer 2015;54:177-184.

4 Boikos SA, Pappo AS, Killian JK, et al. Molecular subtypes of KIT/PDGFRA wild-type gastrointestinal stromal tumors: a report from the National Institutes of Health Gastrointestinal Stromal Tumor Clinic. JAMA Oncol 2016;2:922-928.

5 Gasparotto D, Rossi S, Polano M, et al. Quadruplenegative GIST is a sentinel for unrecognized neurofibromatosis type 1 syndrome. Clin Cancer Res 2017;23: 273-282.

6 Corless CL. Gastrointestinal stromal tumors: what do we know now? Mod Pathol 2014;27(Suppl 1):S1-16.

7 Doyle LA, Hornick JL. Gastrointestinal stromal tumours: from KIT to succinate dehydrogenase. Histopathology 2014;64:53-67.
8 Shi E, Chmielecki J, Tang CM, et al. FGFR1 and NTRK3 actionable alterations in "Wild-Type" gastrointestinal stromal tumors. J Transl Med 2016;14:339.

9 Miettinen M, Fetsch JF, Sobin LH, et al. Gastrointestinal stromal tumors in patients with neurofibromatosis 1: a clinicopathologic and molecular genetic study of 45 cases. Am J Surg Pathol 2006;30:90-96.

10 Mussi C, Schildhaus HU, Gronchi A, et al. Therapeutic consequences from molecular biology for gastrointestinal stromal tumor patients affected by neurofibromatosis type 1. Clin Cancer Res 2008;14: 4550-4555.

11 Paschou M, Doxakis E. Neurofibromin 1 is a miRNA target in neurons. PLoS ONE 2012;7:e46773.

12 Chan JK, Ip YT, Cheuk W. The utility of immunohistochemistry for providing genetic information on tumors. Int J Surg Pathol 2013;21:455-475.

13 Doyle LA, Nelson D, Heinrich MC, et al. Loss of succinate dehydrogenase subunit B (SDHB) expression is limited to a distinctive subset of gastric wild-type gastrointestinal stromal tumours: a comprehensive genotype-phenotype correlation study. Histopathology 2012;61:801-809.

14 Patil DT, Ma S, Konishi M, et al. Utility of BRAF V600E mutation-specific immunohistochemistry in detecting BRAF V600E-mutated gastrointestinal stromal tumors. Am J Clin Pathol 2015;144:782-789.

15 Ritterhouse LL, Barletta JA. BRAF V600E mutationspecific antibody: a review. Semin Diagn Pathol 2015; 32:400-408.

16 Rossi S, Sbaraglia M, Dell'Orto MC, et al. Concomitant KIT/BRAF and PDGFRA/BRAF mutations are rare events in gastrointestinal stromal tumors. Oncotarget 2016;7:30109-30118.

17 Reuss DE, Habel A, Hagenlocher C, et al. Neurofibromin specific antibody differentiates malignant peripheral nerve sheath tumors (MPNST) from other spindle cell neoplasms. Acta Neuropathol 2014;127: 565-572.

18 Röhrich M, Koelsche C, Schrimpf D, et al. Methylationbased classification of benign and malignant peripheral nerve sheath tumors. Acta Neuropathol 2016;131: 877-887.

19 Brenca M, Rossi S, Polano M, et al. Transcriptome sequencing identifies ETV6-NTRK3 as a gene fusion involved in GIST. J Pathol 2016;238:543-549.

20 Andersson J, Sihto H, Meis-Kindblom JM, et al. NF1-associated gastrointestinal stromal tumors have unique clinical, phenotypic, and genotypic characteristics. Am J Surg Pathol 2005;29:1170-1176.

21 Agaimy A, Vassos N, Croner RS. Gastrointestinal manifestations of neurofibromatosis type 1 (Recklinghausen's disease): clinicopathological spectrum with pathogenetic considerations. Int J Clin Exp Pathol 2012;5:852-862.

22 Dombi E, Baldwin A, Marcus LJ, et al. Activity of selumetinib in neurofibromatosis type 1-related plexiform neurofibromas. N Engl J Med 2016;375: 2550-2560.

23 Liu XY, Gerges N, Korshunov A, et al. Frequent ATRX mutations and loss of expression in adult diffuse astrocytic tumors carrying IDH1/IDH2 and TP53 mutations. Acta Neuropathol 2012;124:615-625.

24 Wiestler B, Capper D, Holland-Letz T, et al. ATRX loss refines the classification of anaplastic gliomas and identifies a subgroup of IDH mutant astrocytic tumors with better prognosis. Acta Neuropathol 2013;126: 443-451. 
25 Laycock-van Spyk S, Thomas N, Cooper DN, et al. Neurofibromatosis type 1-associated tumours: their somatic mutational spectrum and pathogenesis. Hum Genomics 2011;5:623-690.

26 McGillicuddy LT, Fromm JA, Hollstein PE, et al. Proteasomal and genetic inactivation of the NF1 tumor suppressor in gliomagenesis. Cancer Cell 2009;16: 44-54.

27 Hollstein PE, Cichowski K. Identifying the ubiquitin ligase complex that regulates the NF1 tumor suppressor and Ras. Cancer Discov 2013;3:880-893.

28 Rossi S, Gasparotto D, Toffolatti L, et al. Molecular and clinicopathologic characterization of gastrointestinal stromal tumors (GISTs) of small size. Am J Surg Pathol 2010;34:1480-1491.

29 Wagner AJ, Remillard SP, Zhang YX, et al. Loss of expression of SDHA predicts SDHA mutations in gastrointestinal stromal tumors. Mod Pathol 2013;26:289-294.

30 Daston MM, Ratner N. Neurofibromin, a predominantly neuronal GTPase activating protein in the adult, is ubiquitously expressed during development. Dev Dyn 1992;195:216-226.

31 Daston MM, Scrable H, Nordlund M, et al. The protein product of the neurofibromatosis type 1 gene is expressed at highest abundance in neurons, Schwann cells, and oligodendrocytes. Neuron 1992;8:415-428. 